\title{
Influência da posição canguru no sistema cardiopulmonar de prematuros em uma Unidade de Terapia Intensiva Neonatal na Amazônia
}

Influence of kangaroo position on cardiopulmonary system of preterm babies in a neonatal intensive care unit in Amazonia

Influencia de la posición del canguro en el sistema cardiopulmonar de los recién nacidos prematuros en una unidad de cuidados intensivos neonatales en la Amazonía

Milene Ribeiro Duarte Sena ${ }^{1 *}$, Patricia Barbosa Ferrarini ${ }^{1}$, Silvia Frare ${ }^{1}$, Valdenira dos Santos Menezes da Cunha ${ }^{2}$, Rodrigo Luis Ferreira da Silva ${ }^{3}$.

\section{RESUMO}

Objetivo: Analisar o efeito da posição canguru (PC) no sistema cardiopulmonar dos recém-nascidos (RN) prematuros internados em uma Unidade de Terapia Intensiva Neonatal (UTIN) paraense. Métodos: Trata-se de um estudo de campo, a amostra do estudo foi composta por $11 \mathrm{RN}$ prematuros com idade gestacional inferior a 37 semanas e com baixo peso, colocados na PC uma vez ao dia durante 60 minutos por três dias consecutivos, onde foram coletados os sinais vitais: frequência cardíaca $(F C)$, frequência respiratória (FR), saturação periférica de oxigênio (SpO2), temperatura ( $\mathrm{T}$ ), nos minutos 0,30 e 60 e Boletim SilvermanAndersen (BSA) antes e após a PC. A intervenção ocorreu no período de agosto a outubro de 2019. Resultados: Foi observada a variação dos resultados, revelando um aumento significativo da temperatura corpórea dos prematuros durante a PC, atingindo uma mediana de $36,8^{\circ}$. Nas demais variáveis, BSA, FC, FR e SpO2, não houveram modificações estatísticas significativas. Conclusão: Conclui-se que a PC promoveu aumento na temperatura corporal dos RNs prematuros, onde foi observado um melhor controle térmico, essencial para a termo regulação dos prematuros.

Palavras-chave: Sinais vitais, Método canguru, Fisioterapia.

\section{ABSTRACT}

Objective: To analyze the effect of kangaroo position (CP) on the cardiopulmonary system of preterm newborn infants (NBs) admitted to a Neonatal Intensive Care Unit (NICU) paraense. Methods: This is a field study held at NICU, sample consisted of 11 preterm infants under 37 weeks of gestation and underweight, placed in CP once a day for 60 minutes for three consecutive days, where vital signs were collected: heart rate (HR), respiratory rate $(\mathrm{RR})$, peripheral oxygen saturation (SpO2), temperature $(\mathrm{T})$ at minutes 0,30 and 60 and Silverman-Andersen Bulletin (BSA) before and after CP. The intervention took place from August to October 2019. Results: The results varied, revealing a significant increase in body temperature of preterm infants during $\mathrm{CP}$, reaching a median of $36.8^{\circ}$. In the other variables, $\mathrm{BSA}, \mathrm{HR}, \mathrm{FR}$ and $\mathrm{SpO} 2$, there were no significant statistical changes. Conclusion: It was concluded that CP promoted an increase in body temperature of premature infants, where a better thermal control was observed, essential for the term regulation of premature infants.

Key words: Vital signs, Kangaroo method, Physiotherapy.

\footnotetext{
1 Instituto Esperança de Ensino Superior (IESPES), Santarém - PA *E-mail: milenefisio04@hotmail.com

${ }^{2}$ Hospital Regional do Baixo Amazonas (HRBA), Santarém - Pará.

3 Universidade do Estado do Pará (UEPA), Santarém - Pará.
} 


\section{RESUMEN}

Objetivo: analizar el efecto de la posición canguro (PC) en el sistema cardiopulmonar de recién nacidos prematuros (NB) ingresados en una Unidad de Cuidados Intensivos Neonatales (UCIN) paraense. Métodos: Este es un estudio de campo celebrado en la UCIN, la muestra del estudio consistió en 11 recién nacidos prematuros menores de 37 semanas de gestación y bajo peso, colocados en CP una vez al día durante 60 minutos por tres días consecutivos, donde se recolectaron signos vitales: frecuencia cardíaca (FC), frecuencia respiratoria (RR), saturación periférica de oxígeno (SpO2), temperatura ( $T$ ) en los minutos 0,30 y 60 y Silverman-Andersen Bulletin (BSA) antes y después de la CP. La intervención tuvo lugar de agosto a octubre de 2019. Resultados: Los resultados variaron, revelando un aumento significativo en la temperatura corporal de los bebés prematuros durante la PC, alcanzando una mediana de $36.8^{\circ}$. En las otras variables BSA, HR, FR y SpO2, no hubo cambios estadísticos significativos. Conclusión: Se concluyó que la PC promovió un aumento en la temperatura corporal de los recién nacidos prematuros, donde se observó un mejor control térmico, esencial para el término regulación de los recién nacidos prematuros.

Palabras clave: Signos vitales, Método canguro, Fisioterapia.

\section{INTRODUÇÃO}

$\mathrm{Na}$ atualidade, os índices de nascimentos prematuros por ano, são de 20 milhões. A qual comumente está associada ao baixo peso, que segundo o Ministério da Saúde (MS), são os recém-nascidos (RN) com menos de 2,500g. Já os prematuros são aqueles nascidos com menos de 37 semanas (BRASIL, 2018). O recémnascido prematuro (RNP) necessita de um suporte avançado de cuidados e observações rigorosas para manutenção da vida por meio de sua permanência em uma Unidade de Terapia Intensiva Neonatal (UTIN), sendo este um local que proporciona um alto nível de desenvolvimento de estresse ao RNP, devido as condições físicas do ambiente que normalmente apresentam ruídos advindos dos equipamentos e da equipe multiprofissional; iluminação excessiva que impossibilita o prematuro de diferenciar o dia e a noite; toque repetitivo para manuseio da postura e procedimentos invasivos, dolorosos e estímulos térmicos variados (NASCIMENTO MHM, 2012).

Segundo Panceri C, et al. (2012) as principais causas do nascimento prematuro estão correlacionadas à alguns transtornos ou injúrias que aceleram este processo e suas consequências oferecem riscos ao desenvolvimento neuropsicomotor, como complicações e alterações aos RN prematuros nos seguintes marcos: controle postural, motricidade fina, motricidade grossa, visão, linguagem, audição, cognição e homeostasia, que interferem na qualidade de vida do RNP e influenciam no estado emocional e psicológico de seus familiares.

A probabilidade de prematuros obterem um melhor prognóstico vem aumentando, devido à maior oferta de tecnologias nos serviços públicos de média e alta complexidade e a crescente humanização na resolutividade dos problemas de saúde, o que geram benefícios ao desenvolvimento do RNP (PANCERI C, et al., 2012). Dentre as áreas da fisioterapia, o fisioterapeuta desempenha papel fundamental na UTIN para os RN prematuros que são influenciados pelo posicionamento que é indispensável no decorrer dos primeiros dias de vida, favorecendo condições satisfatórias na hemodinâmica e no desenvolvimento motor.

A humanização, por sua vez, compreende um novo modelo de atenção aos RNP pais e familiares inseridos na UTIN, respeitando suas características e individualidades. O atendimento humanizado visa minimizar condutas agressivas, que se caracterizam pela atuação profissional, aliadas pelo toque e segurança técnica durante a execução de procedimentos em seu atendimento (BRASIL, 2014).

Em 5 de Julho de 2000, o Ministério da Saúde lançou por meio da portaria № 693, a Norma de Atenção Humanizada ao Recém-Nascido de Baixo Peso (Método Canguru). Somente em 12 de julho de 2007 entrou em vigor pela portaria № 1683, a Norma de Orientação para Implantação do Método Canguru. As UTINs que já haviam implementado o método, apenas mantiveram ou realizaram novas adaptações nos atendimentos prestados pela equipe multiprofissional (BRASIL, 2014). Dentre a equipe multiprofissional destaca-se a 
fisioterapia por intervir de forma integral nos cuidados e necessidades dos RNP. A atuação do fisioterapeuta é de suma importância devido os conhecimentos prévios de posicionamento mãe-bebê adequado à postura na posição canguru (PC), além de ter competência para repasse e preparo dos familiares na aplicabilidade das técnicas referentes à posição. Como consequência o RNP aprenderá movimentos nos quais estará interagindo com o ambiente externo facilitando a socialização e adaptação (PINTO M, et al., 2008).

Conforme Azevedo VMGO, et al. (2017), o fisioterapeuta utiliza a posição canguru como uma estimulação multimodal nas UTIN, sendo destacados: estímulo tátil através do toque familiar e contato pele a pele; estimulação vestibular devido a mudanças de posicionamentos e acomodações do RNP; estimulação auditiva através da voz materna e dos sons emitidos pela caixa torácica da mãe ou familiar; estimulação olfatória produzida pela essência materna e o processo da produção de leite; e a estimulação gustativa inteiramente através da amamentação.

O método canguru surgiu na Colômbia em 1979, pelos Dr. Reys Sanabria e Dr. Hector Martine, com o propósito de redução dos altos índices de RNP internados nas UTIN, tempo de internação e de custos hospitalares, como modelo de assistência perinatal promovendo o contato pele a pele entre mãe-bebê, beneficiando o vínculo afetivo, favorecendo a estabilidade térmica e possibilitando melhor desenvolvimento, através dos cuidados de humanização e métodos de intervenção biopsicossocial (SANTOS MH e AZEVEDO FILHO FM, 2016).

Azevedo VMGO, et al. (2017), indagam em seu estudo que a posição canguru deve ser iniciada desde o momento da internação do RNP, realizada com cuidado humanizado começando com o toque das mãos no RNP e progredir para a posição canguru, permanecendo em média cinquenta a sessenta minutos na posição, com o objetivo de regulação da temperatura, estabilidade fisiológica e comportamental, regulagem do sono e descanso, crescimento e desenvolvimento neurológico, alivio de dores e desconforto do RN.

O RNP é posicionado de frente na vertical sobre o tórax do familiar sem roupas e acessórios vestindo apenas a bata, e o RNP utilizando somente a fralda para realizar o contato pele a pele, os membros superiores (MMSS) e membros inferiores (MMII) ficam semi-flexionados, a cabeça fica voltada para um dos lados na linha média e envolto pela bata do familiar na posição canguru (AZEVEDO VMGO, et al., 2017).

A posição canguru pode proporcionar grandes estímulos no processo hemodinâmico do RNP, além de ser de baixo custo, fácil aplicabilidade em qualquer UTIN, possibilitado variações benéficas nos sinais vitais dos RN prematuros, temperatura $(T)$, saturação (SpO2), frequência respiratória ( $F R$ ), frequência cardíaca $(F C)$ e bem-estar, contribuindo para a fisioterapia como meio adicional de protocolos de atendimentos nas UTIN (OLMEDO MD, et al., 2012).

Nesse sentido, este estudo se propõe a analisar o efeito da posição canguru no sistema cardiopulmonar dos RN internados em uma UTIN, propondo enriquecimento de informações para os pesquisadores, além de gerar conhecimentos e discussões na instituição sobre o tema, bem como, apresentar os resultados aos profissionais do setor da UTIN, a fim de contribuir com a rotina do atendimento multiprofissional.

\section{MÉTODOS}

O estudo foi realizado em uma UTIN paraense. A amostra foi composta por $11 \mathrm{RN}$ prematuros de ambos os sexos e com baixo peso. A intervenção para a coleta de dados ocorreu no período de agosto a outubro de 2019.

Foram incluídos neste estudo RN prematuros de ambos os sexos com idade gestacional inferior a 37 semanas, com peso abaixo de 2.500 gramas, com prescrição de fisioterapia no prontuário e aceitação dos familiares a participarem do estudo voluntariamente. Foram excluídos os participantes que por algum motivo interromperam o protocolo, excederam o tempo determinado, solicitaram desligamento da pesquisa, passaram por processo cirúrgico durante o período do protocolo, apresentaram instabilidade hemodinâmica, com restrições para mudança de decúbito, com distúrbios respiratórios graves, extremo baixo peso no momento da intervenção e RN prematuros com complicações cardíacas. Os participantes foram selecionados 
conforme os critérios de inclusão e exclusão previamente selecionados, posteriormente, o familiar foi convidado a participar do estudo mediante contato pessoal, onde foram esclarecidos sobre a posição canguru. Em seguida, ocorreu a leitura e assinatura do Termo Consentimento Livre e Esclarecido (TCLE), no qual os participantes foram informados quanto a garantia de preservação do anonimato e da autonomia para se retirar da pesquisa a qualquer momento.

Para determinar a amostra do estudo, foi utilizado um protocolo para coleta de dados na posição canguru, composto por identificação do RNP e do familiar, avaliação do desconforto respiratório do RNP por meio da escala Boletim de Silverman-Andersen (BSA), no momento anterior e posterior a intervenção, padrão muscular respiratório e padrão postural. Em seguida, o RNP era retirado do berço ou incubadora usando apenas a fralda, colocado no colo do familiar na posição canguru vertical, com a cabeça lateralizada, os membros superiores e inferiores flexionados e aduzidos próximos ao tronco e em contato direto com a pele do tórax do responsável, que estava usando apenas uma bata que envolvia o RNP oferecendo maior segurança e conforto aos participantes.

Seguindo o protocolo da posição canguru, os sinais vitais foram coletados em três momentos diferentes: início - minuto 0; durante - minuto 30 , final - minuto 60, conforme protocolo adaptado de Olmedo MD, et al. (2012), no qual foi coletado a frequência cardíaca $(\mathrm{FC})$ e saturação periférica de oxigênio $\left(\mathrm{SpO}_{2}\right)$ por meio de um oxímetro de pulso colocado na extremidade do membro inferior do RNP, frequência respiratória (FR) aferida pela ausculta pulmonar e a temperatura $(T)$ aferida por meio de termômetro digital. Ao final da intervenção, o RNP era devolvido ao berço ou incubadora na posição de melhor conforto e realizado o toque gentil e humanizado pela pesquisadora responsável do estudo.

Os dados coletados por meio das variáveis dos sinais vitais foram digitalizados, tabulados e analisados pelo programa Microsoft Excel ${ }^{\circledR} 2013$ e BioEstat ${ }^{\circledR}$ 5.3, onde iniciou-se com a estatística descritiva na qual obtivemos os valores calculados, como: mínimo, máximo, média e desvio padrão das variáveis: $\mathrm{FC}, \mathrm{FR}, \mathrm{SpO}_{2}$ e T. Foi também realizada a estatística inferencial, onde foram analisadas comparações entre as variáveis por meio dos seguintes testes: Shapiro-wilk para avaliar a normalidade do conjunto dos dados, Friedman para obter os valores de comparação entre as variáveis dos sinais vitais, Wilcoxon para calcular a variável do BSA, gerando dados quantitativos para o estudo, sendo o valor usado para a significância de $p \leq 0,05$.

O estudo seguiu os princípios éticos presentes nas Normas de Pesquisa Envolvendo Seres Humanos, descritos na Resolução No 466/12 do Conselho Nacional de Saúde (CNS). Sendo submetido ao Comitê de Ética em Pesquisa com Seres Humanos (CEP) do Instituto Esperança de Ensino superior (IESPES), CAAE 11007019.4.0000.8070, aprovado pelo parecer $n^{\circ} 3.381 .257$, em 10 de junho de 2019.

\section{RESULTADOS}

Foram avaliados 11 (onze) RN prematuros com idade gestacional inferior a 37 semanas e com baixo peso, sendo 5 (cinco) do sexo feminino e 6 (seis) do sexo masculino, conforme demonstrado abaixo (Tabela 1).

Tabela 1 - Peso ao nascer, sexo e idade gestacional dos RN prematuros avaliados.

\begin{tabular}{ccccccc}
\multicolumn{8}{c}{ Peso ao nascer $(\mathrm{Kg})$} \\
Sexo & $\mathbf{N}$ & Mínimo & Máximo & Média & Mediana & Desvio Padrão \\
F & 5 & 0.885 & 2.34 & 1.54 & 1.55 & 0.4693 \\
M & 6 & 0.85 & 2.27 & 1.38 & 1.28 & 0.4762 \\
\hline \multicolumn{7}{c}{ Idade Gestacional (Semanas) } \\
Sexo & $\mathbf{N}$ & Mínimo & Máximo & Média & Mediana & Desvio Padrão \\
F & 5 & 29 & 35 & 31.80 & 31 & 2.0396 \\
M & 6 & 27 & 34 & 30.33 & 30.5 & 2.1344 \\
\hline
\end{tabular}

Fonte: Sena MRD, et al., 2019. 
Conforme dados demostrados na tabela 1, observa-se que a mediana de peso para RNs prematuros do sexo feminino foi de $1.55 \mathrm{~kg}$, contudo para o sexo masculino de $1.28 \mathrm{~kg}$. A idade gestacional resultou em 31 semanas para sexo feminino e 30.5 semanas para o sexo masculino.

Os RN prematuros avaliados pelo BSA no início e ao final da intervenção não apresentaram valores estatísticos significativos de desconforto respiratório, onde o valor de significância $p=0.1913$ (Tabela 2).

Tabela 2 - Desconforto respiratório avaliado pelo Boletim Silverman - Andersen.

\begin{tabular}{ccc}
\hline & BSA & \\
\hline \multirow{2}{*}{ Inicial / Final } & $\mathbf{Z}-$ Teste & 1.3066 \\
& $\mathbf{p}$ Valor & 0.1913 \\
\hline
\end{tabular}

Legenda:

BSA: Boletim Silverman - Andersen; Z - Teste: valor referente ao teste estatístico; $p$ Valor: valor de significância.

Fonte: Sena MRD, et al., 2019.

A análise estatística do estudo observou a variação dos resultados, revelando um aumento significativo da temperatura corpórea dos $\mathrm{RN}$ prematuros durante a intervenção na posição canguru. Nas demais variáveis como FC, FR, SpO2 não houveram modificações estatísticas significativas (Tabela 3).

Tabela 3 - Sinais vitais no início - minuto 0, durante - minuto 30 e final - minuto 60, na intervenção da Posição Canguru.

\begin{tabular}{ccccccc}
\hline Sinais Vitais & & Mínimo & Máximo & Mediana & Desvio Padrão & p valor \\
\hline \multirow{3}{*}{ T } & Minuto 0 & 35.1 & 39.5 & 36.5 & 0.6913 & 0.0086 \\
& Minuto 30 & 35.1 & 37.6 & 36.5 & 0.4479 & 0.0261 \\
& Minuto 60 & 35.8 & 37.7 & 36.8 & 0.379 & 0.2888 \\
\multirow{2}{*}{ SpO2 } & Minuto 0 & 78 & 100 & 97 & 4.2402 & 0.0087 \\
& Minuto 30 & 91 & 100 & 96 & 2.8776 & 0.0106 \\
& Minuto 60 & 85 & 100 & 96 & 3.4281 & 0.0092 \\
& & & & & & \\
& Minuto 0 & 35 & 64 & 48 & 6.7336 & 0.9007 \\
& Minuto 30 & 32 & 64 & 49 & 7.1272 & 0.3211 \\
& Minuto 60 & 25 & 64 & 49 & 6.4997 & 0.9817 \\
& & & & & & 0.4946 \\
& Minuto 0 & 107 & 186 & 154 & 19.1515 & 0.9583 \\
& Minuto 30 & 105 & 196 & 149 & 19.4988 & 0.3493 \\
\hline
\end{tabular}

\section{Legenda:}

FC: frequência cardíaca; $\mathrm{FR}$ : frequência respiratória; $\mathrm{SpO}_{2}$ : saturação periférica de oxigênio; $\mathrm{T}$ : temperatura; $p$ valor: valor de significância.

Fonte: Sena MRD, et al., 2019. 
Conforme observado, os valores da temperatura variaram de acordo com a mediana de 36.5 no minuto 0 , mantendo o mesmo valor no minuto 30 , elevando-se para $36.8 \mathrm{em} 60$ minutos de acordo com o protocolo de intervenção da posição canguru. No entanto, a mediana das variáveis de FC, FR, SpO2 não demostraram valores estatisticamente significativos na comparação entre minuto 0 e minuto 60 de intervenção do protocolo, sendo que os testes inferenciais utilizados exigem que $p$ seja menor que 0,05 para que haja diferença significativa entre os valores.

O estudo não apresentou diferenças significativas entre os $1^{\circ}, 2^{\circ}$ e $3^{\circ}$ dias de intervenção nas variáveis de FC, FR, SpO2. Considerando para esse cálculo a significância entre os valores aferidos antes, durante e após a aplicação do método, sendo $p=0.8659 ; p=0.602 ; p=0.934$, respectivamente. No entanto, apresentou diferença significativa na variável temperatura, onde $p<0.0001$.

\section{DISCUSSÃO}

O estudo apresenta uma proporção de 5 (45\%) RNP do sexo feminino e 6 (55\%) do sexo masculino, diferentemente do estudo de Ramos HAC e Cuman RKN (2009), que apresentou em seus resultados uma amostra de $106 \mathrm{RN}$ prematuros, sendo 55 do sexo feminino e 51 do sexo masculino. Como demonstrado no estudo de Lamy Filho F, et al. (2008) que apresenta uma amostra de $616 \mathrm{RN}$ prematuros que estiveram na posição canguru, prevalecendo 316 do sexo feminino e 300 do sexo masculino. Corroborando com o presente estudo, Miltersteiner AR, et al. (2003) apresenta uma amostra de 23 RNs prematuros, sendo que o sexo masculino prevaleceu com $15 \mathrm{RNs}$ prematuros e 8 do sexo feminino, entretanto este estudo mostra predomínio de nascimentos de RN prematuros do sexo masculino, porém a questão de gênero não é significativa.

$\mathrm{Na}$ análise do peso ao nascer foi encontrada uma mediana de $1.55 \mathrm{~kg}$ para o sexo feminino e $1.28 \mathrm{~kg}$ para o sexo masculino, com idades gestacionais de 31 semanas para sexo feminino e 30.5 semanas para sexo masculino, entretanto observa-se que o sexo feminino se destaca em relação do peso ao nascer e idade gestacional com resultados estatísticos superiores. Nobre RG, et al. (2017) observou em seu estudo que após intervenção no método canguru com $78 \mathrm{RN}$ prematuros, houve um aumento na média de peso adquirido ao dia, assim contribuindo para melhora do baixo peso ao nascer destes prematuros. Em relação a idade gestacional máxima do estudo foi de 35 e a mínima de 27 semanas, em contrapartida o estudo de Miltersteiner AR, et al. (2003), apresentou uma idade gestacional máxima de 37 e a mínima de 24 semanas, contudo a idade gestacional não demostrou interferência com relação a aplicação da posição canguru nos $\mathrm{RN}$ prematuros.

Em observação as variáveis do BSA para o desconforto respiratório dos RN prematuros, não houveram resultados significativos entre os três dias de intervenção, mas notou-se melhora parcial a curto prazo onde alguns RN prematuros que iniciavam o posicionamento com pontuação 2 reduziam para 1 ou 0 no decorrer do protocolo. Em contrapartida, o estudo de Lanza FC, et al. (2012) notou redução estatisticamente significante do BSA após a colocação do paciente em decúbito ventral, isso pode ser explicado pela alteração do posicionamento de ventral para vertical, onde o mesmo acompanha os movimentos torácicos do familiar, adquirindo relaxamento do RNP e também o estudo de Ludington-Hoe SM, et al. (2011) também verificou melhora no padrão respiratório e diminuição de gemência após a intervenção na posição canguru.

Com relação à temperatura corporal dos RNP, este estudo demostra resultados benéficos, onde houve aumento significativo da temperatura corporal dos $\mathrm{RN}$ prematuros durante a intervenção na posição canguru, no minuto 30 e ao final, no minuto 60, o mesmo pode-se notar no estudo de Almeida CM, et al. (2007) realizado com 22 RN prematuros de baixo peso, que avaliou a temperatura após 30 minutos de intervenção, notando aumento significativo do valor da temperatura, atribuindo assim o benefício deste aumento ao contato pele a pele e troca de calor com o familiar, ofertado por meio do posicionamento na posição canguru. O presente estudo também concorda com Payne $\mathrm{H}$, et al. (2019), onde mostrou que a temperatura se eleva para acompanhar as mudanças de calor do responsável, que está em contato direto com o RNP. Além do posicionamento adotado pelo RNP, assumindo uma posição flexora, permitindo assim uma conservação do 
calor comparado a quando está colocado na incubadora ou berço. A posição canguru favorece a estabilização referente aos parâmetros fisiológicos dos $\mathrm{RN}$ prematuros, proporcionado pelo contato direto pele a pele e vínculo familiar.

Conforme estudo de Almeida CM, et al. (2007), demonstra-se a grande dificuldade de manutenção da temperatura corpórea dos $\mathrm{RN}$ prematuros, em razão à falta de sudorese, produção defeituosa de calor pelo baixo peso e pouca movimentação, além da imaturidade dos centros reguladores da temperatura. Tratandose da retirada do RNP da incubadora ou berço pode-se notar a baixa da temperatura, onde a hipotermia prologada aumenta o consumo de energia e oxigênio na tentativa de manutenção do calor corpóreo, o que é prejudicial ao RNP. Deste modo nota-se a importância da fisioterapia na aplicabilidade da posição canguru para a manutenção da troca de calor, sabendo-se que o controle térmico adequado para prosseguir no tratamento fisioterapêutico é de suma importância (ALMEIDA CM, et al., 2007).

Notando-se a relação entre a temperatura e oxigênio podemos observar a diminuição do calor, levando ao aumento do consumo de oxigênio, assim comprometendo a hemodinâmica do RNP, que neste estudo não foram observadas variáveis quantitativas na intervenção de 3 dias consecutivos em consideração a SpO2, no entanto notou-se que houve melhora na oxigenação tecidual em comparação do minuto 0 ao minuto 60 , deste modo não gerando resultados significativos à longo prazo, corroborando com o estudo de Olmedo MD, et al. (2012) que notou a melhora na SpO2 a curto prazo após a posição canguru, mas não gerando resultados estatisticamente significativos durante os três dias de intervenção.

Em contrapartida, a pesquisa de Tenório EAM, et al. (2010) relata um aumento significativo na SpO2, podendo ser elucidado devido a condição confortável e relaxante do RNP durante e após aplicação da posição canguru, devido a diminuição de consumo de oxigênio, melhorando a SpO2 periférica do RNP.

Neste estudo não houve diferença significativa em relação a variável FR antes, durante e após a posição canguru, discordando de OLMEDO MD, et al. (2012) onde observou-se a diminuição da FR na posição canguru, o que se leva em consideração as condições anatômicas e fisiológicas dos RNs prematuros, que em contato pele a pele, o RNP tende a acompanhar a respiração fisiológica do responsável, dessa forma levando a diminuição e condições favoráveis ao RNP.

O presente estudo concorda com Miltersteiner AR, et al. (2003) que realizou uma pesquisa com 22 bebês pré-termos submetidos a posição canguru, onde não foram encontrados resultados significativos para FR, mas os autores evidenciaram que os RN prematuros mantem a estabilidade da FR decorrente da diminuição de apneias ou pausas respiratórias enquanto permanece na vertical em contato pele a pele com o responsável durante a intervenção na posição canguru.

Em relação a FC este estudo não apontou dados estatísticos significativos dentro das variáveis, concordando com o estudo de Almeida CM, et al. (2007), que não encontraram variáveis satisfatórias. Contudo o aumento da FC pode estar relacionado devido o manuseio do RNP sendo retirado da incubadora ou berço para realização da posição canguru, aumentando o estresse consequentemente aumentando a FC, por outro lado, temos a diminuição da FC que pode estar relacionada com o estado de tranquilidade, segurança e sono do RNP durante a posição canguru, proporcionando melhora no bem-estar e qualidade de vida, mas vale ressaltar que são dados incerto pois diante da fisiologia do RN prematuro os dados podem ser sobrepostos pelos padrões do responsável. Azevedo VMGO, et al. (2017) evidenciou em sua revisão bibliográfica, autores que demonstraram aumento significativo da FC, porém mantendo dentro dos parâmetros de normalidade.

\section{CONCLUSÃO}

Conclui-se por meio dos resultados estatísticos que a posição canguru promoveu aumento da temperatura corporal dos RNs prematuros, observando um melhor controle térmico. Este controle contribui para os protocolos de atendimentos executados pela equipe multiprofissional, principalmente aqueles voltados para técnicas de posicionamento terapêutico, habilidade desenvolvida pelo fisioterapeuta. Contudo, o posicionamento canguru favorece alterações fisiológicas benéficas para o RNP de baixo peso, tendo em vista 
que o estudo foi realizado por apenas três dias consecutivos. Desta forma, sugere-se que a posição canguru seja incentivada nas UTIN, pois é uma posição simples, de baixo custo e eficaz, e ainda que novos estudos sejam realizados a fim de fortalecer a relevância desta temática.

\section{AGRADECIMENTOS E FINANCIAMENTO}

Agradecimento ao Hospital Regional do Baixo Amazonas Dr. Waldemar Penna por incentivar a pesquisa científica disponibilizando seus campos de prática para a intervenção como a UTIN, fortalecendo a produção científica no Oeste do Pará, ao professor Dr. Rodrigo Luis Ferreira da Silva por colaborar com seus conhecimentos para nossa pesquisa, à Dra. Valdenira dos Santos Menezes da Cunha pelo incentivo a aplicabilidade da posição canguru nos RNP da UTIN, à orientadora Milene Ribeiro Duarte Sena pelo incentivo e apoio, sendo a base do conhecimento para desenvolvimento deste estudo e ao Instituto Esperança de Ensino Superior (IESPES) pela excelente qualidade oferecida na formação superior no interior da Amazônia.

\section{REFERÊNCIAS}

1. ALMEIDA CM, et al. Efeitos do Método Mãe Canguru nos Sinais Vitais de Recém-Nascidos Pré-Termo de Baixo Peso. Revista Brasileira de Fisioterapia. 2007; 11(1): 1-5, ISSN 1413-3555.

2. AZEVEDO VMGO, et al. Cuidado mãe canguru em recém-nascidos pré-termo sob suporte ventilatório: avaliação dos estados comportamentais. Revista Brasileira Saúde Maternidade Infantil, 2011; 11(2): 133-138.

3. AZEVEDO VMGO, et al. Efeitos da posição canguru no sistema cardiorrespiratório de recém-nascidos pré-termo. Programa de Atualização em Fisioterapia Pediátrica e Neonatal: Cardiorrespiratória e Terapia Intensiva - PROFISIO. 2017; 1(6): 119-49.

4. BRASIL, Ministério da Saúde. Atenção à Saúde do Recém-Nascido. Guia para os Profissionais de Saúde. 2018; v. 4.

5. BRASIL, Ministério da Saúde. Secretaria de Atenção à Saúde. Departamento de Ações Programáticas Estratégicas. Atenção humanizada ao recém-nascido de baixo peso: Método Canguru: caderno do tutor. Departamento de Ações Programáticas Estratégicas. 2014; 1. ed.

6. CABRAL LA, et al. Estratégias favorecedoras do desenvolvimento neuropsicomotor de recém-nascidos pré-tremo: da UTI ao ambulatório de seguimento. PROFISIO, 2015; (4): 95-127.

7. DEFILIPO ÉC, et al. Kangaroo position: Immediate effects on the physiological variables of preterm and low birth weight newborns. Fisioter Mov,2017; 30(1): 219-27, ISSN 1980-5918.

8. HENNIG MAS, et al. Atenção humanizada ao recém-nascido de baixo-peso. Método Canguru e cuidado centrado na família: correspondências e especificidades. Revista de Saúde Coletiva,2010; 20(3): 835-852.

9. JOHNSTON, Cíntia et al. I Recomendação brasileira de fisioterapia respiratória em unidade de terapia intensiva pediátrica e neonatal. Artigo Especial, Departamento de Fisioterapia - AMIB - Brasil, 2012; 24 (2)119-129.

10. LAMY FILHO F, et al. Evaluation of the neonatal outcomes of the kangaroo mother method in Brazil. Jornal de Pediatria, 2008; 84(5): 428-435.

11. LANZA FC, et al. Benefícios do decúbito ventral associado ao CPAP em recém-nascidos prematuros. Fisioterapia Pesquisa. 2012;19(2):135-40.

12. LIMA NDC, et al. Construção e Validação de Conteúdo de Instrumento de Coleta de Dados em Unidade Neonatal. Revista da Rede de Enfermagem do Nordeste, 2009; 10(3): 97-106.

13. LUDINGTON- Hoe SM. Evidence-Based Review of Physiologic Effects of Kangaroo Care. Current Women's Health Reviews. 2011; Aug:7(3):243-53.

14. MADUREIRA KT. Efeitos da Posição Canguru na Resposta Fisiológica e no Estado Comportamental de RecémNascidos Pré-Termo de Muito Baixo Peso em Ventilação Mecânica. Dissertação (Pós-Graduação em Ciências da Reabilitação) - Universidade Federal de Minas Gerais (MG), 2010; 80, CDU: 615.8-153.31.

15. MILTERSTEINER AR, et al. Resposta fisiológica da posição mãe-canguru em bebês pré-termo de baixo peso e ventilado espontaneamente. Brasil Saúde Materna Infantil. 2003; 3(4): 447-455.

16. MOURA DS e MAIA J A. Quando a Fisioterapia se Faz Necessária na UTI Neonatal? Revista Saúde Criança Adolescente, 2009; 1(1): $38-40$.

17. NASCIMENTO MHM. Tecnologia para mediar o cuidar-educando no acolhimento de "familiares cangurus" em unidade neonatal: Estudo de Validação. Dissertação (Mestrado em Enfermagem) - Universidade do Estado do Pará (UEPA), Belém-PA, 2012; n.244,172 f, CDD: 21ed. 618.9201. 
18. NETO JAS e RODRIGUES BMRD. Tecnologia como Fundamento do Cuidar em Neonatologia. Artigo enfermagen, $2010 ; 19(2): 372-377$.

19. NOBRE RG, et al. Weight-Gain Velocity in Newborn Infants Managed with the Kangaroo Method and Associated Variables. Journal of Maternal and Child Health, 2017; 21(1): 128-135.

20. OLMEDO MD, et al. Respostas fisiológicas de recém-nascidos pré-termo submetidos ao Metódo Mãe-Canguru e a posição prona. Campo Grande (MS). 2012; 19(2): 115-121.

21. PANCERI C, et al. A influência da hospitalização no desenvolvimento motor de bebês internados no Hospital de Clínicas de Porto Alegre. (UFRGS), Rio Grande do Sul. 2012; 32(2), $8 f$.

22. PAYNE H, et al. Systematic Review Of Kangaroo Care. University of Arkansas-Fort Smith. 2019-09-20T15:53:00Z.

23. PINTO M, et al. Intervenção motora precoce em neonatos prematuros. Universidade Católica do Rio Grande do Sul (HSL-PUCRS), 2008; 1(2): 10p.

24. RAMOS HAC, CUMAN RKN. Fatores de Risco para Prematuridade: Pesquisa Documental. Esc Anna Nery Rev Enferm, 2009; 13 (2): 297-304.

25. REICHERT APS, et al. Humanização do Cuidado da UTI Neonatal - Artigo de Revisão. Revista Eletrônica de Enfermagem, 2007; 09(01): $200-213$.

26. SANTOS DSS e TEIXEIRA EC. Vínculo Mãe-Filho no Contexto da Terapia Intensiva Neonatal: Uma Revisão Sistemática. Faculdade Adventista da Bahia,2017; 1(2): 12.

27. SANTOS MH, AZEVEDO FILHO FM. Benefícios do método Mãe Canguru em recém-nascidos pré-termo ou baixo peso: uma revisão da literatura. Universitas: Ciências da Saúde, Brasília, 2016; 14(1): 67-76.

28. SOUZA AKCM, et al. Ganho de peso em recém-nascidos submetidos ao contato pele a pele. Revista CEFAC, 2018; 20(1): 53-60.

29. TENÓRIO EAM, et al. Avaliação dos parâmetros fisiológicos em recémnascidos pré-termos de baixo peso antes e após a aplicação do método mãe-canguru. Fisioterapia Brasil. 2010; 11(1): 44-48.

30. VENANCIO SI e ALMEIDA H. Método Mãe Canguru: aplicação no Brasil, evidências científicas e impacto sobre o aleitamento materno. Jornal Pediatrico, 2004; 80(5): 173-180, ISSN 0021-7557. 